Supporting Information

\title{
A General Kinetic-Optical Model for Solid-State Reactions Involving the Nano Kirkendall Effect. The Case of Copper Nanoparticle Oxidation
}

Mariano D. Susman, Alexander Vaskevich, "Israel Rubinstein ${ }^{*}$

Department of Materials and Interfaces, Weizmann Institute of Science, Rehovot 7610001, Israel

*Corresponding author. Email: alexander.vaskevich@weizmann.ac.il israel.rubinstein@weizmann.ac.il 


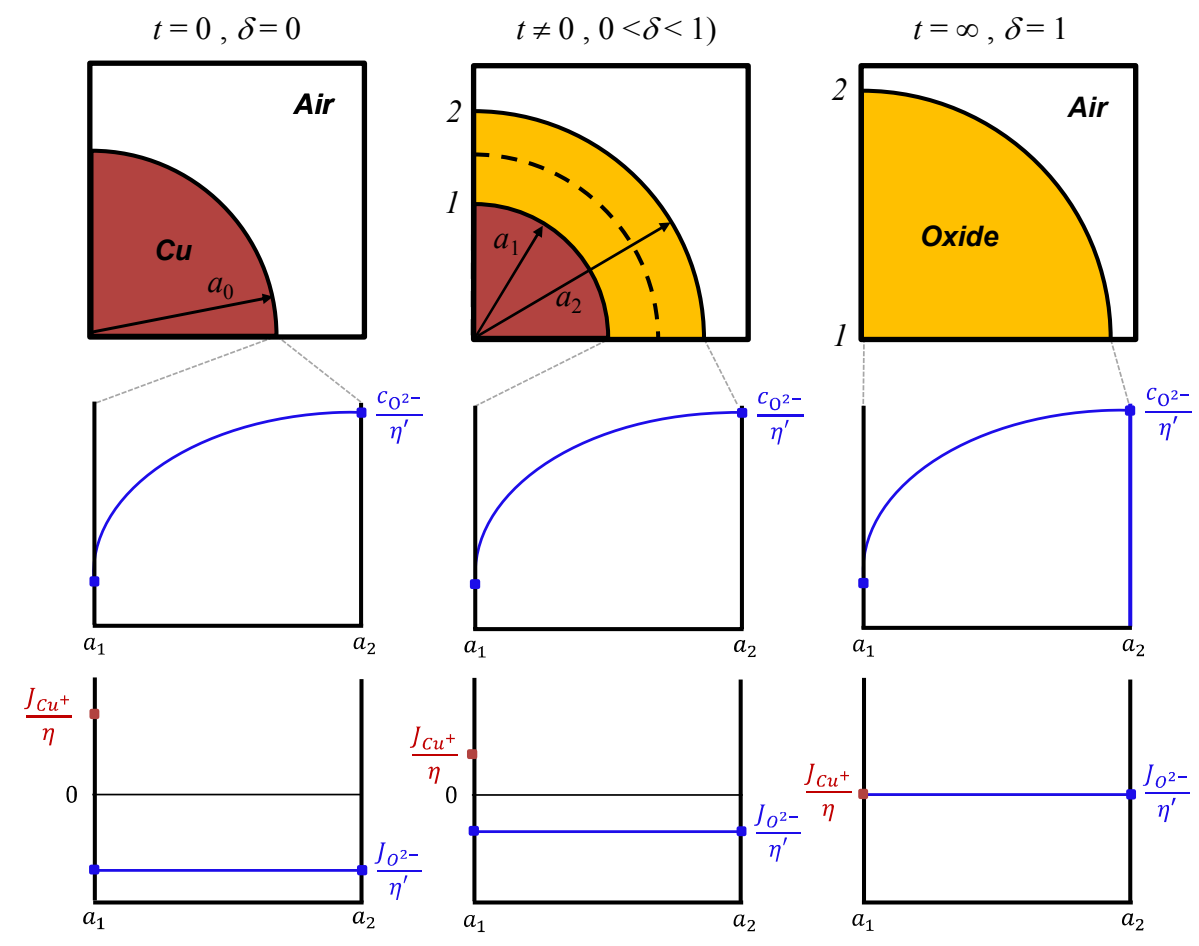

Figure S1. Schematic description of $\mathrm{Cu}_{2} \mathrm{O}$ NP formation according to the Valensi-Carter (VC) model, showing (top) the NP geometric conformation, (middle) the concentration profiles, and (bottom) the species flow, as a function of the time or conversion fraction (progressing from left to right). Full lines indicate the behavior of the species, assumed by the model. 


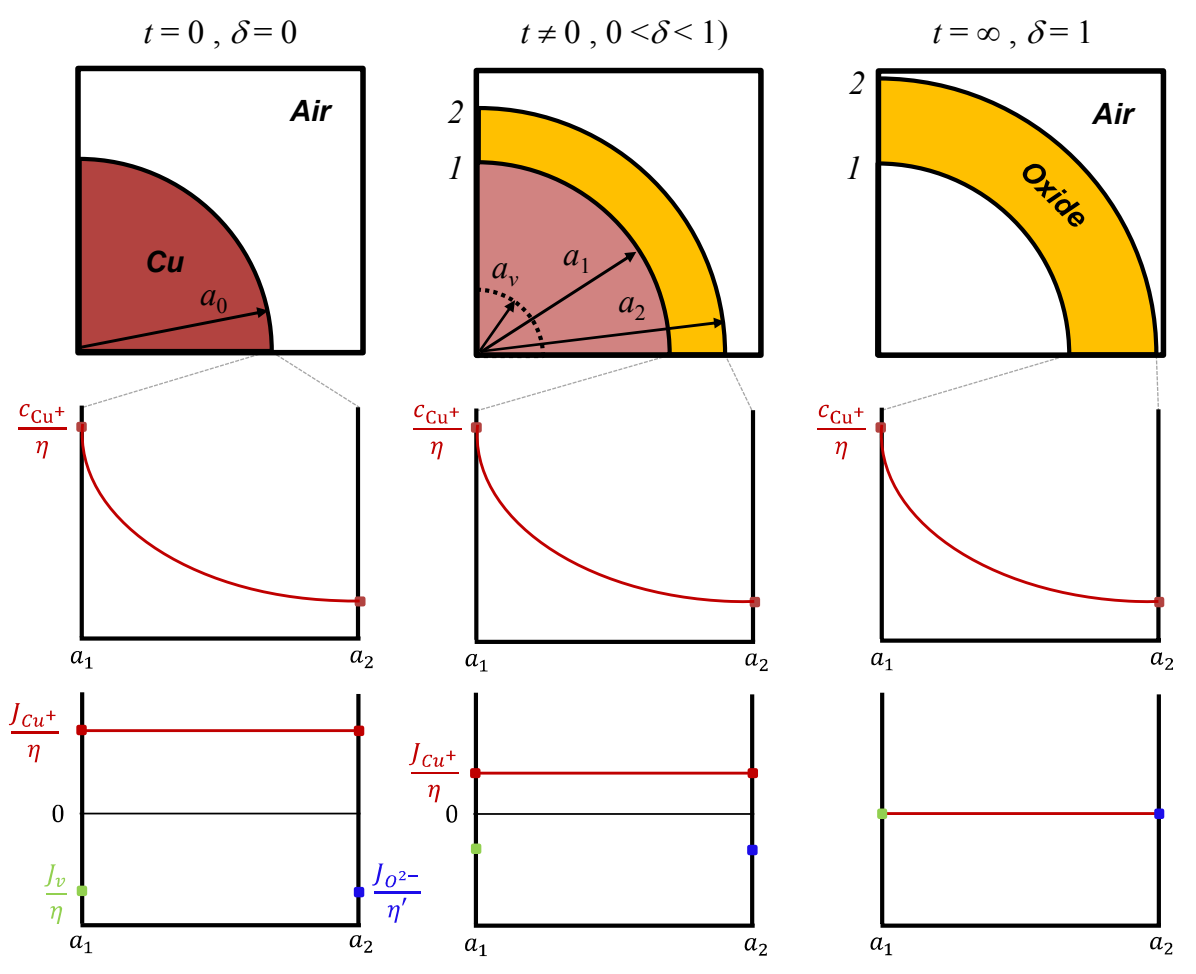

Figure S2. Schematic description of $\mathrm{Cu}_{2} \mathrm{O}$ NP formation according to the nano Kirkenall effect (NKE) model, showing (top) the NP geometric conformation, (medium) the concentration profiles, and (bottom) the species flow, as a function of the time or conversion fraction (progressing from left to right). Full lines indicate the behavior of the species, assumed by the model. Note that the outgoing flow of $\mathrm{Cu}$ is compensated by inward flow of vacancies, as $O^{2-}$ is assumed to negligibly diffuse through the oxide layer.
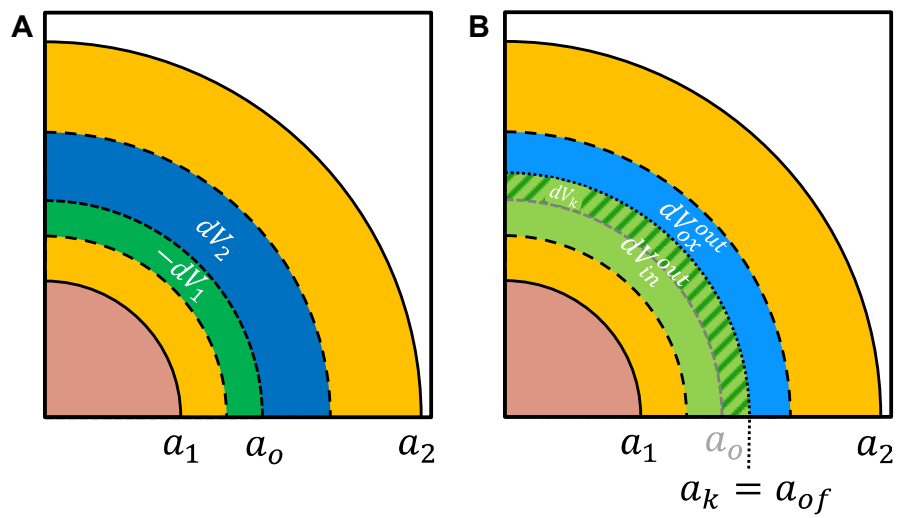

Figure S3. Schematic description of $\mathrm{Cu}_{2} \mathrm{O}$ NP formation observed from (A) a static frame-of-reference centered at $a_{0}$, and (B) a moving oxide lattice frame-of-reference, free to advance with any Kirkendall shift (given by $d V_{k}$ ), which depends on the combination of $K$ and $\phi$ values. The inward and outward growths, actually taking place at the oxide boundaries, are assumed to occur at the Kirkendall surface. For $d V_{k}=0$, scheme $\mathrm{B}$ reduces to scheme A. Note that the oxidation front may be considered to coincide with the Kirkendall surface $\left(a_{o f}=a_{k}\right)$. 


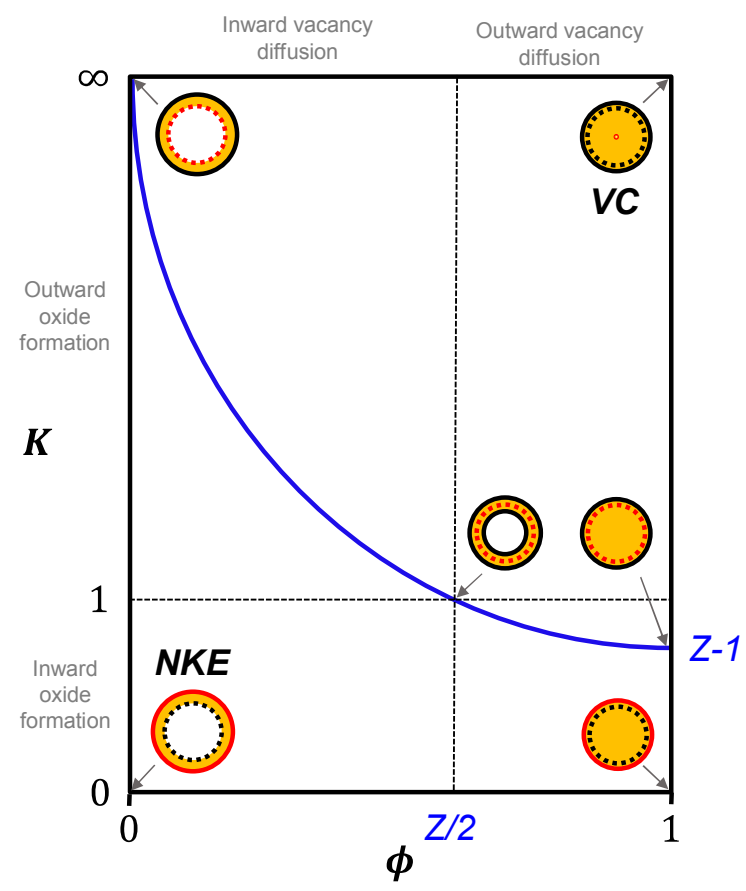

Figure S4. The various oxidation regimes, for different combinations of $K$ and $\phi$ values. Note that the general model accounts for all situations. Restricted cases where no Kirkendall shift occurs $\left(V_{k}=0\right)$ are indicated by the blue line. Red circles indicate the location of the oxidation front (or Kirkendall surface). Dotted circles indicate the location of the initial particle surface (at $a_{0}$ ).

A) $a_{0}=20 \mathrm{~nm}$
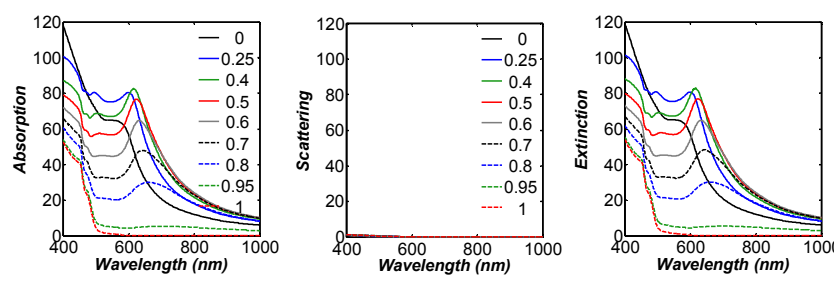

$\phi=1$
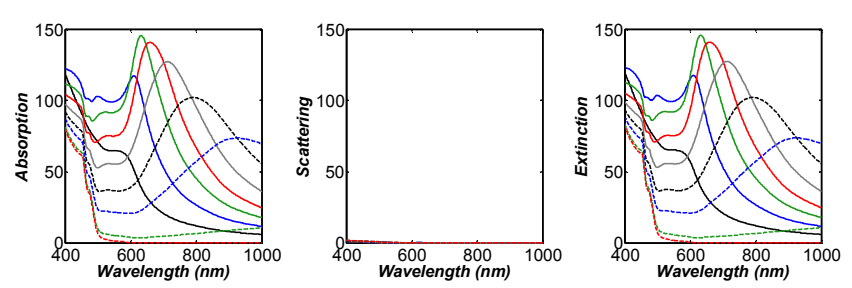

$\phi=0.5$
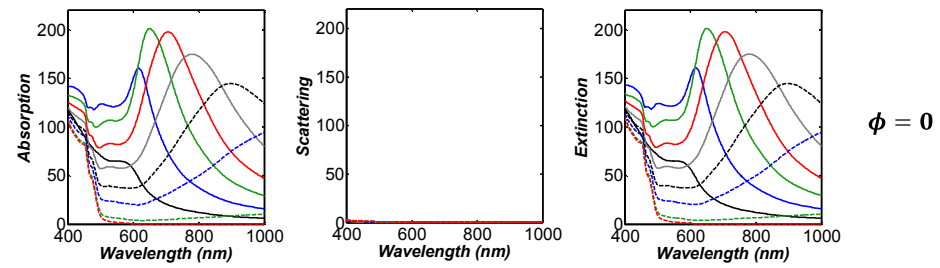
B) $a_{0}=90 \mathrm{~nm}$
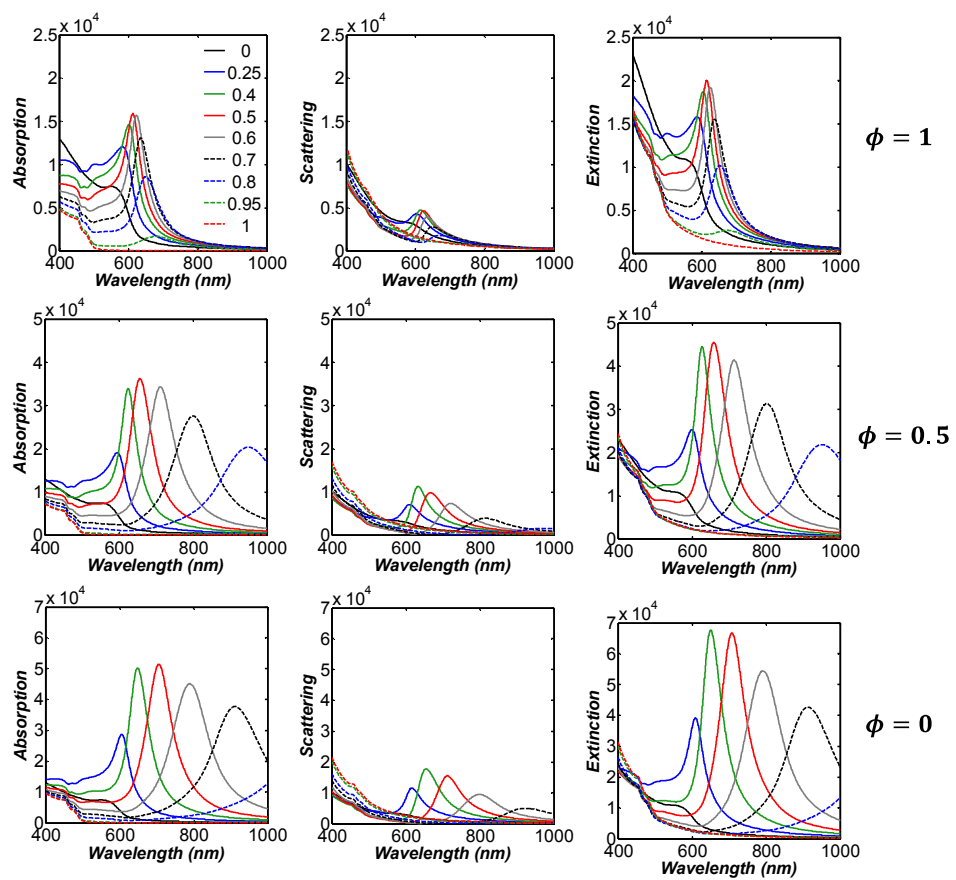

C) $a_{0}=150 \mathrm{~nm}$
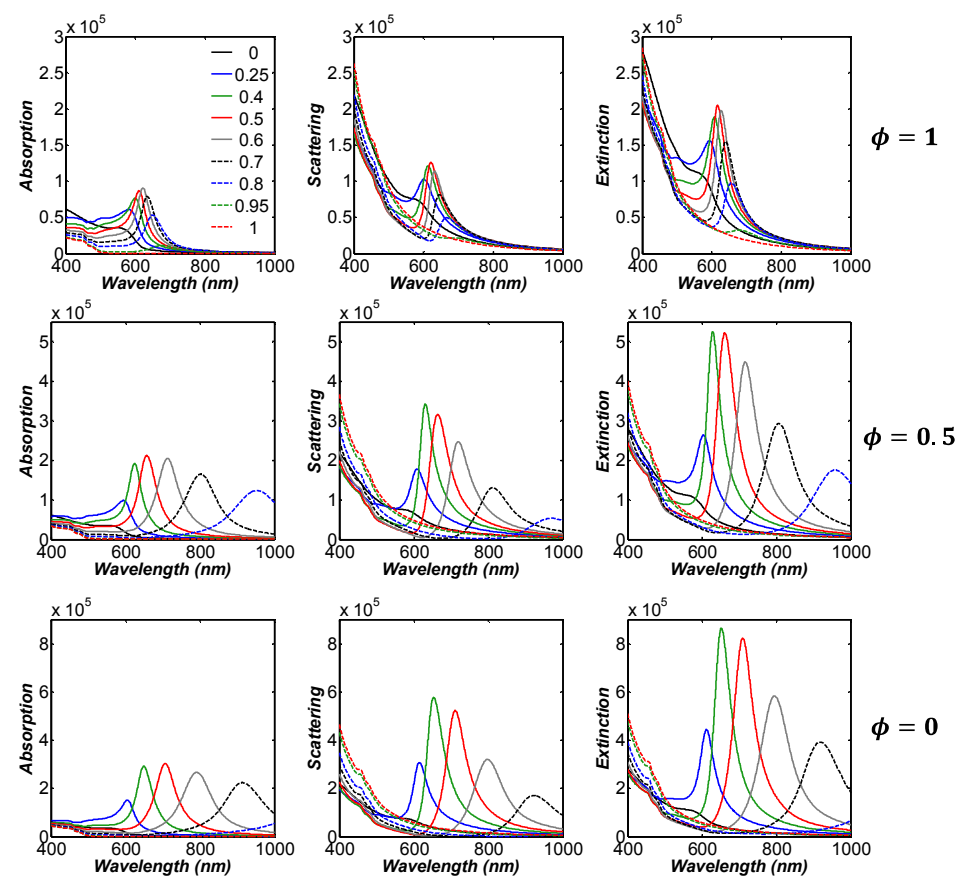

Figure S5. Absorption, scattering and extinction cross-sections for $\mathrm{Cu}$ NPs of $(A) 20,(B) 90$ and $(C)$ $150 \mathrm{~nm}$ initial diameter, as a function of the conversion fraction (from 0 to 1 , indicated), and for different $\phi$ values. 

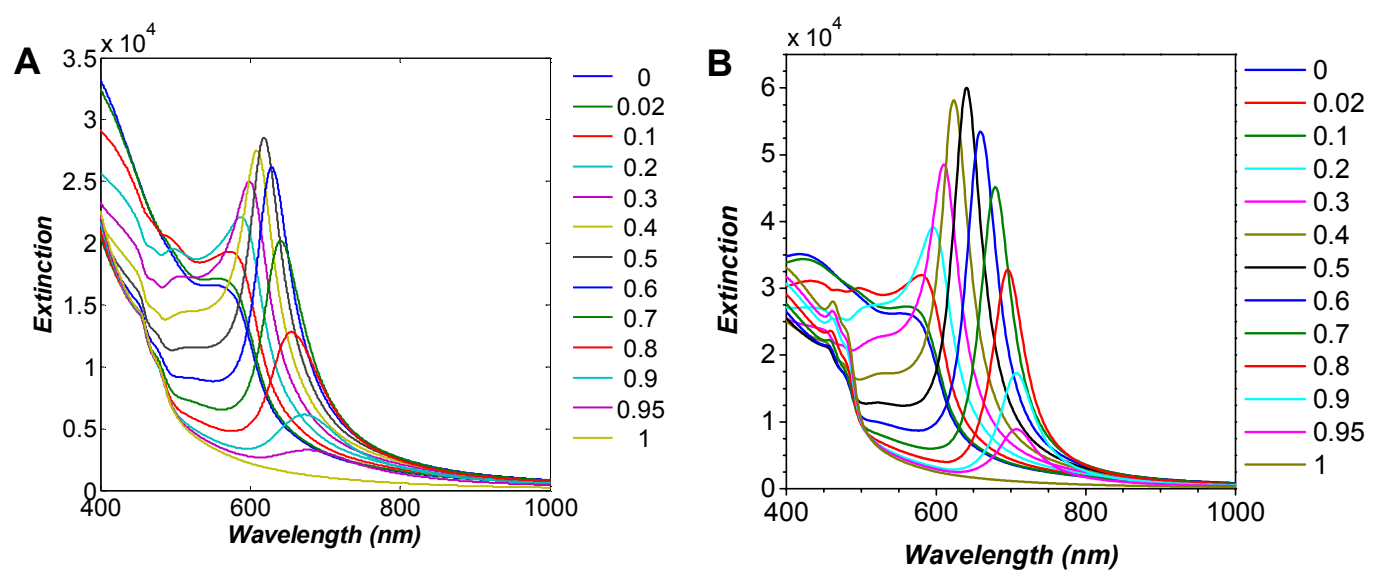

Figure S6. Extinction cross-sections for $\mathrm{Cu}$ NPs of $90 \mathrm{~nm}$ initial diameter undergoing oxidation $(\phi=$ 1 ), as a function of the conversion fraction (from 0 to 1 , indicated). Results obtained with $(A)$ the Matlab script (quasi-static approximation), and with $(B)$ MieLab 0.2.1 software, using the same medium dielectric constant (1.13, corresponding to air/glass supported NPs) and geometric parameters, are presented and can be compared.
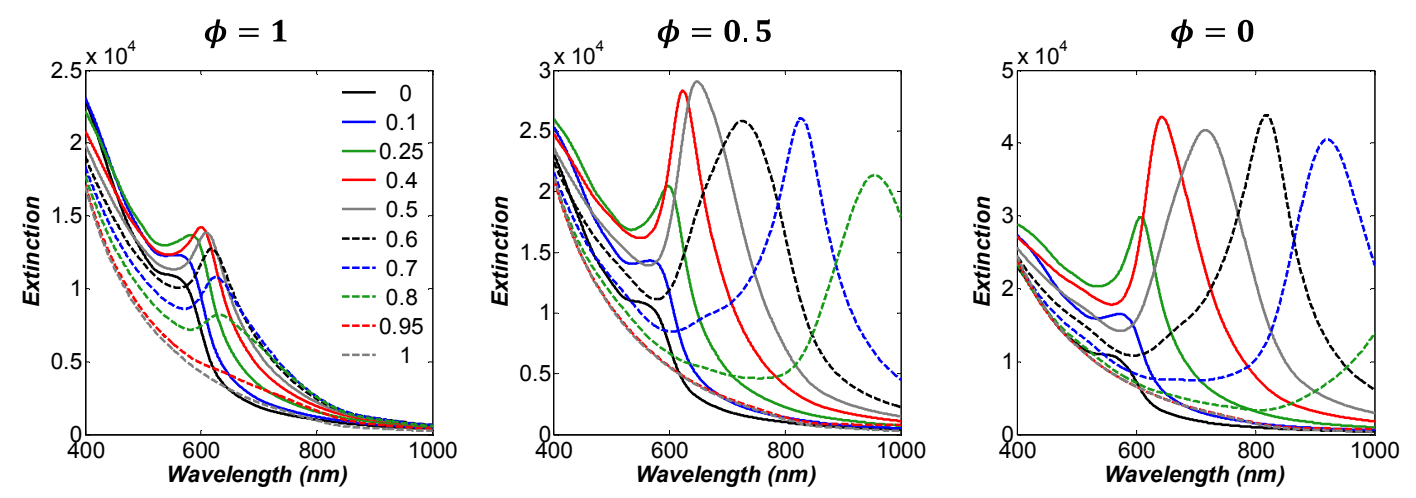

Figure S7. Extinction cross-sections for $\mathrm{Cu}$ NPs of $90 \mathrm{~nm}$ initial diameter undergoing oxidation to $\mathrm{CuO}$, as a function of the conversion fraction (from 0 to 1 , indicated), and for different $\phi$ values. 


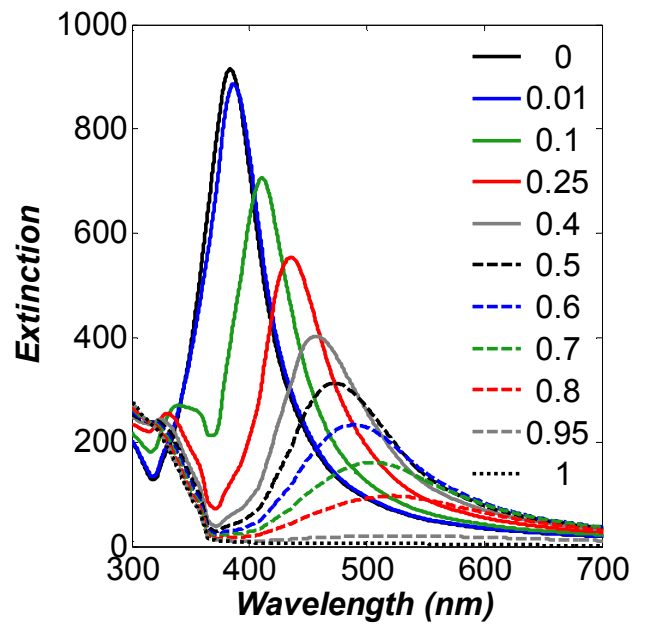

Figure S8. Extinction cross-sections for $20 \mathrm{~nm}$ (initial diameter) Ag NPs undergoing oxidation to $\mathrm{Ag}_{2} \mathrm{O}$ $(\phi=1)$, as a function of the conversion fraction (from 0 to 1 , indicated). 


\section{Matlab script}

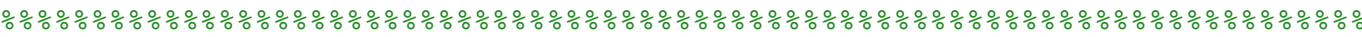

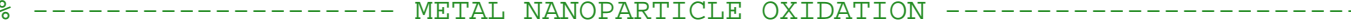
ㅇ------- Mie solutions for core-shell spherical nanoparticles ----------\%--------------- under the quasi-static approximation ------------------\%-- General kinetic model considering the NKE, without Kirkendall shift -\%---------------- Calculations as a function of time

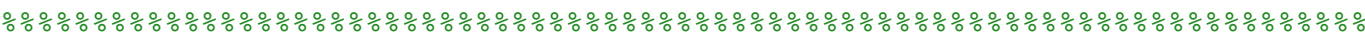

\% In this script, spherical metal NPs with a given normal size distribution o react according to a general kinetic model, where the NKE is considered.

- The standard deviation of the distribution can

\% be set for the particular size distribution in the system under study.

\% Bulk properties of the materials have to be defined (density and formula

\% weight), as well as the stoichiometric coefficients of the reaction.

\% The parameters phi and $k$, have to be set in the script for defining the

\% extent of core contraction and the reaction rate.

- A Mie scattering model (in the quasi-static approximation) of spherical

\% core-shell particles was used to model the overall optical behavior of

\% the evolving core-shell NP system.

\% An Effective Medium Approximation (EMA) (Maxwell-Garnett approximation)

\% was used to model the optical properties of the core under vacancy

o accumulation.

\% A Kreibig's correction is applied for the size dependent metal dielectric

o constant (relevant at the low size limit). Plasma frequency,

\% Fermi velocity, and bulk damping constant have to be set.

$\%$

\% The files containing the bulk dielectric constants of metal (1) and oxide

\% (2) have to be in the format: 1st column: Wavelength (nm), 2nd column: n,

\% 3rd column: k, separated by tabulations.

$\circ$

\% Calculations are performed as a function of the reaction time, according to o the integral reaction model.

응

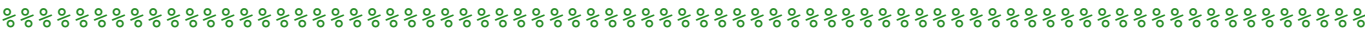

$\mathrm{ClC}$

clear all

close all

lambda $=(400: 0.1: 1000) . '$; $\%$ in $\mathrm{nm}$

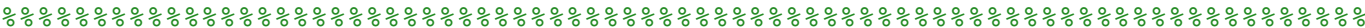
응 PROPERTIES OF THE MATERIALS

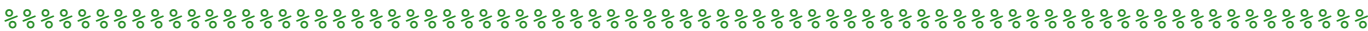

응 Dielectric constant of the core

\% For $\mathrm{Cu}$ : Jonhnson and Christy's bulk data (Cu-JC.txt)

filename='Cu-JC.txt';

ㅇ Select the appropriate core material file.

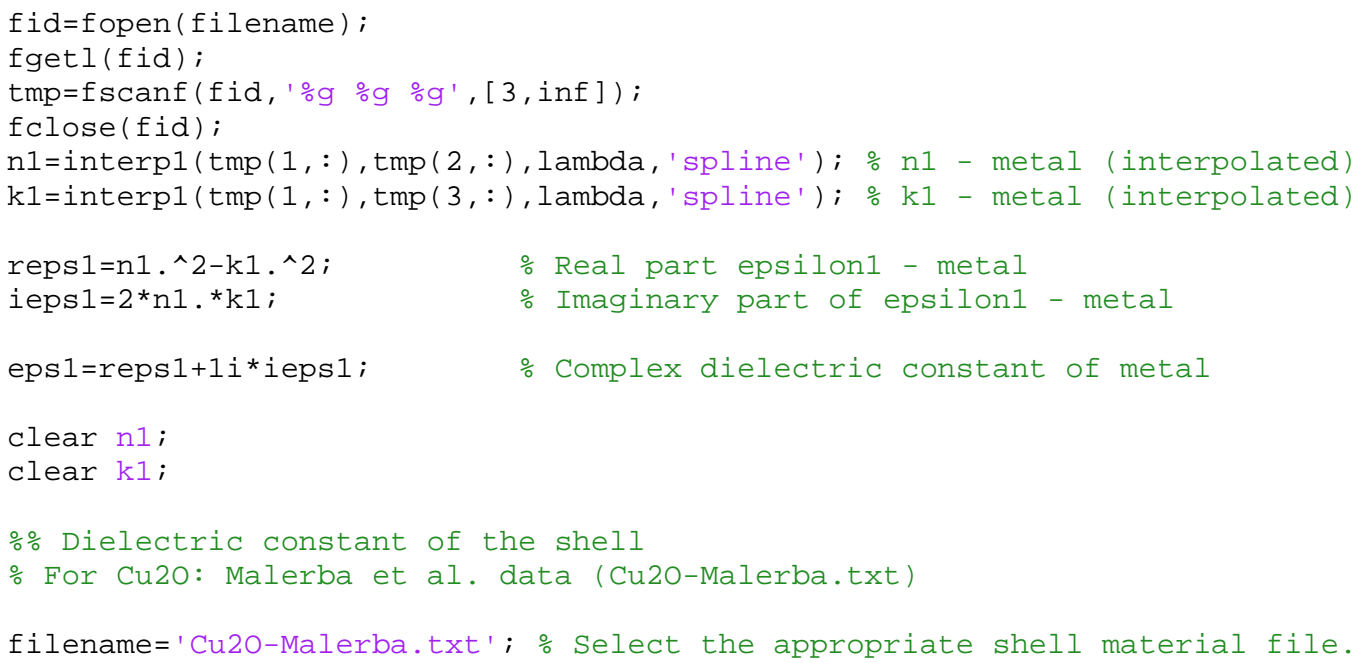




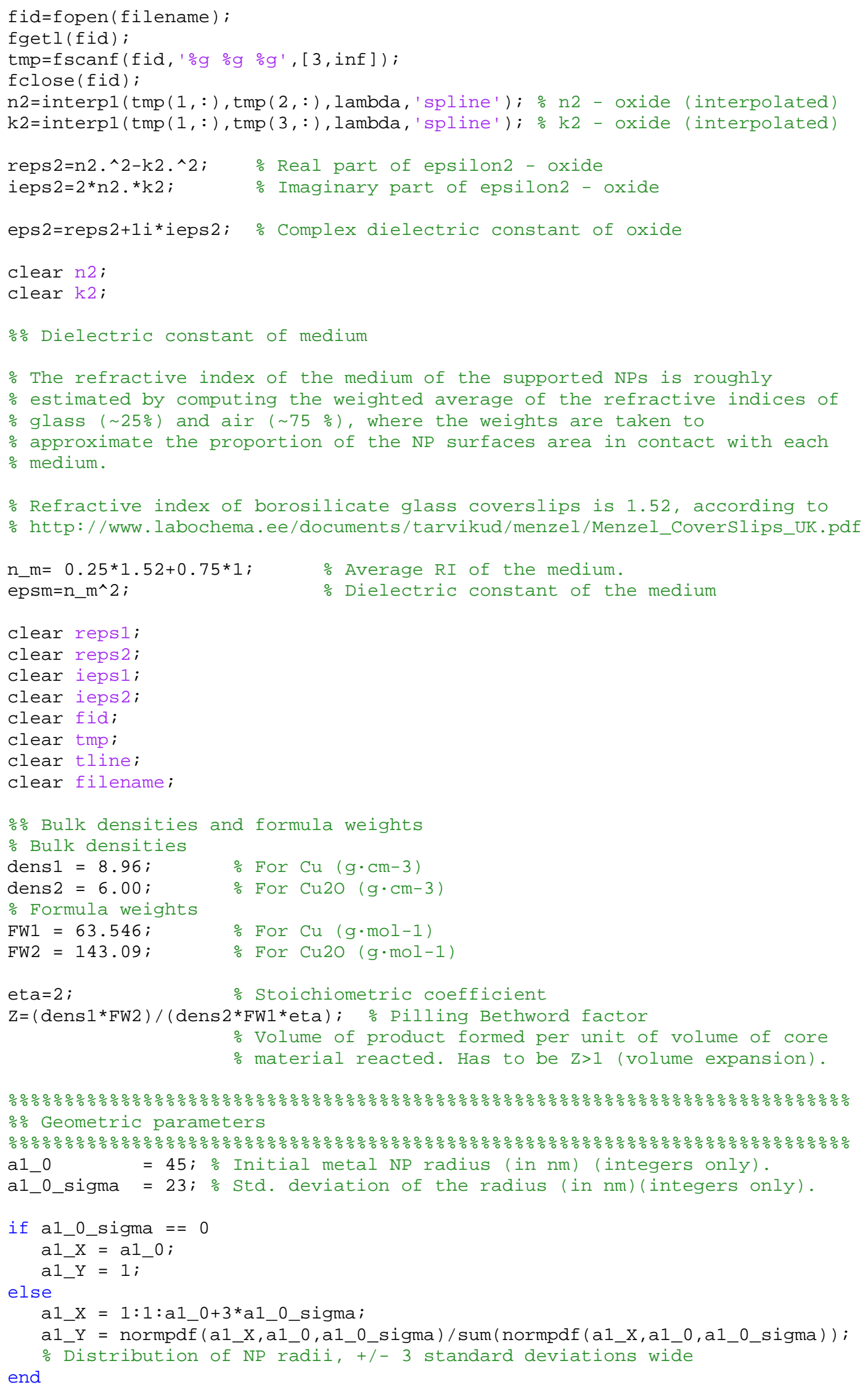

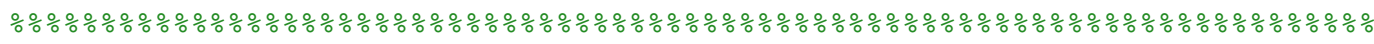
응 General kinetic model

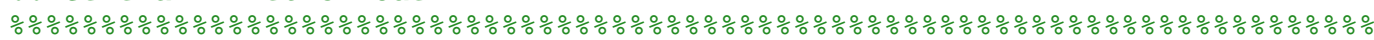




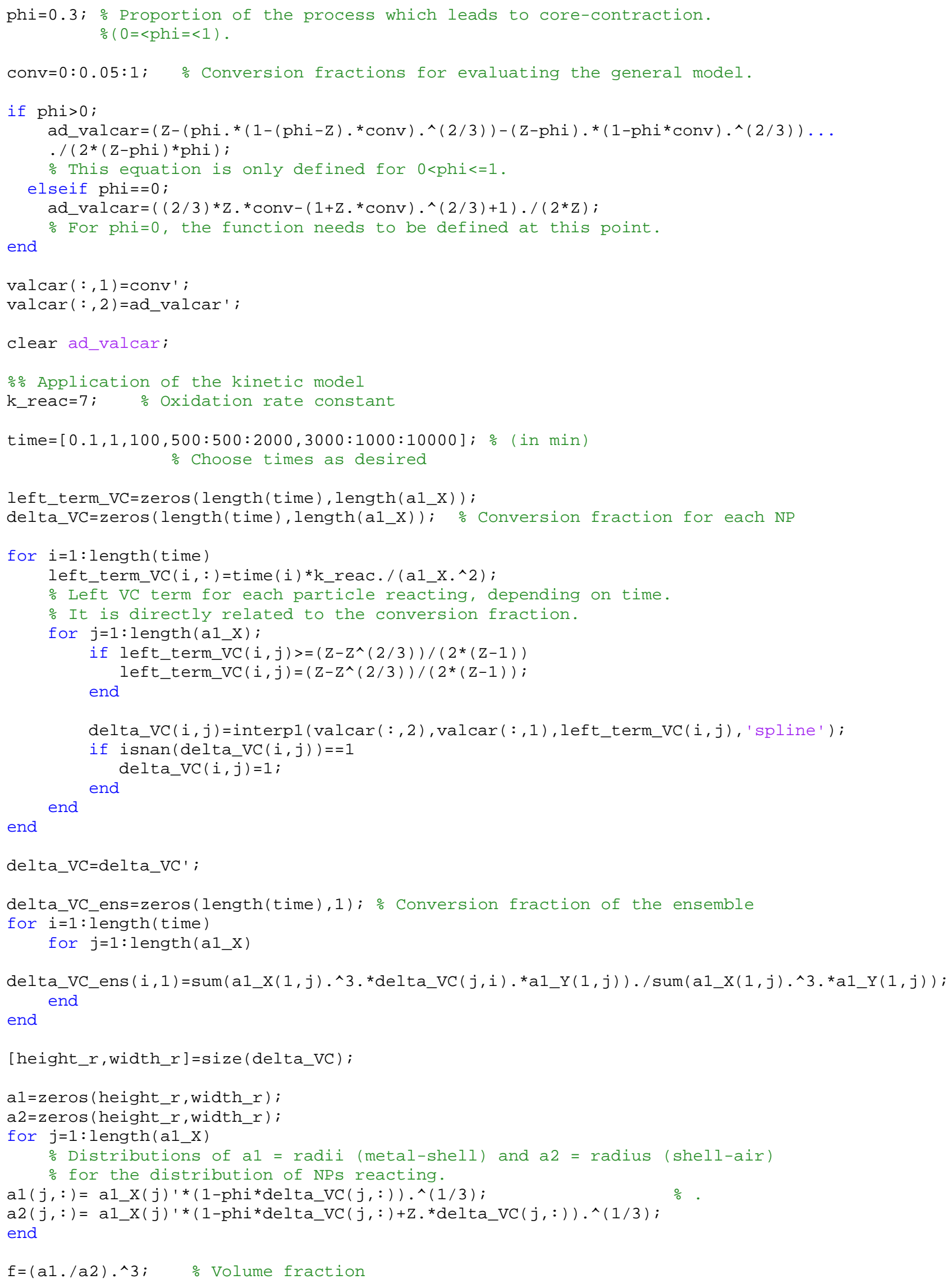




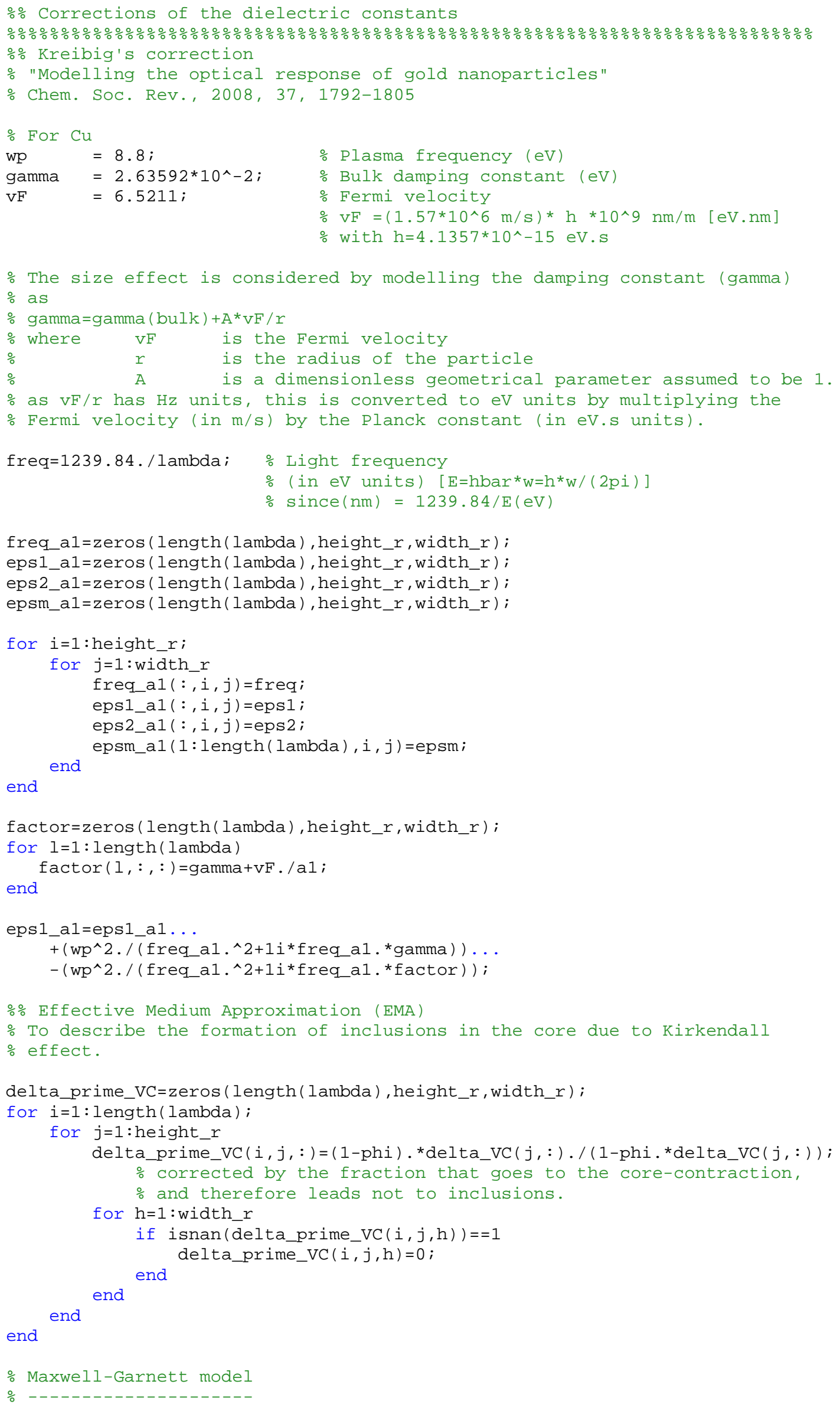




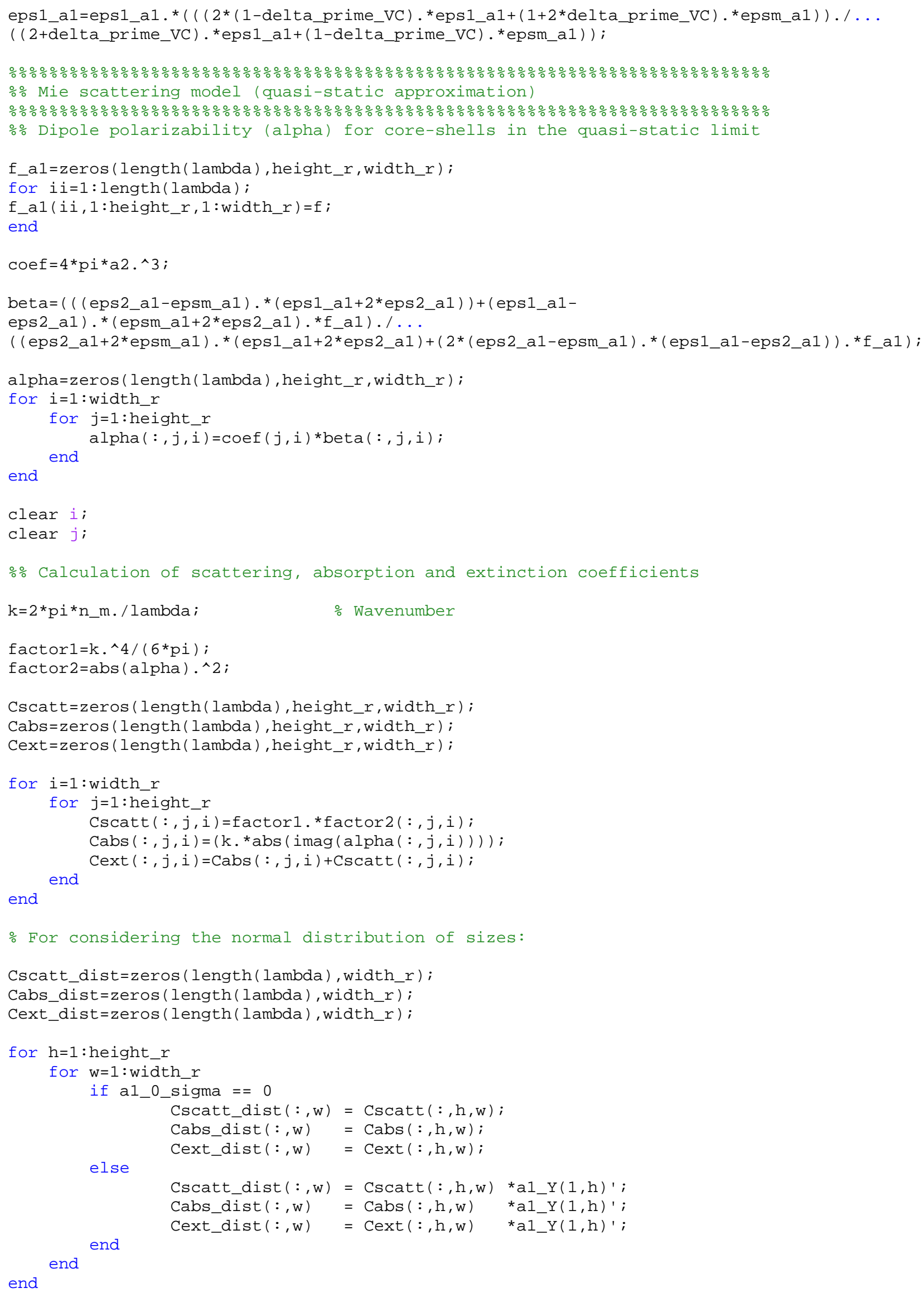




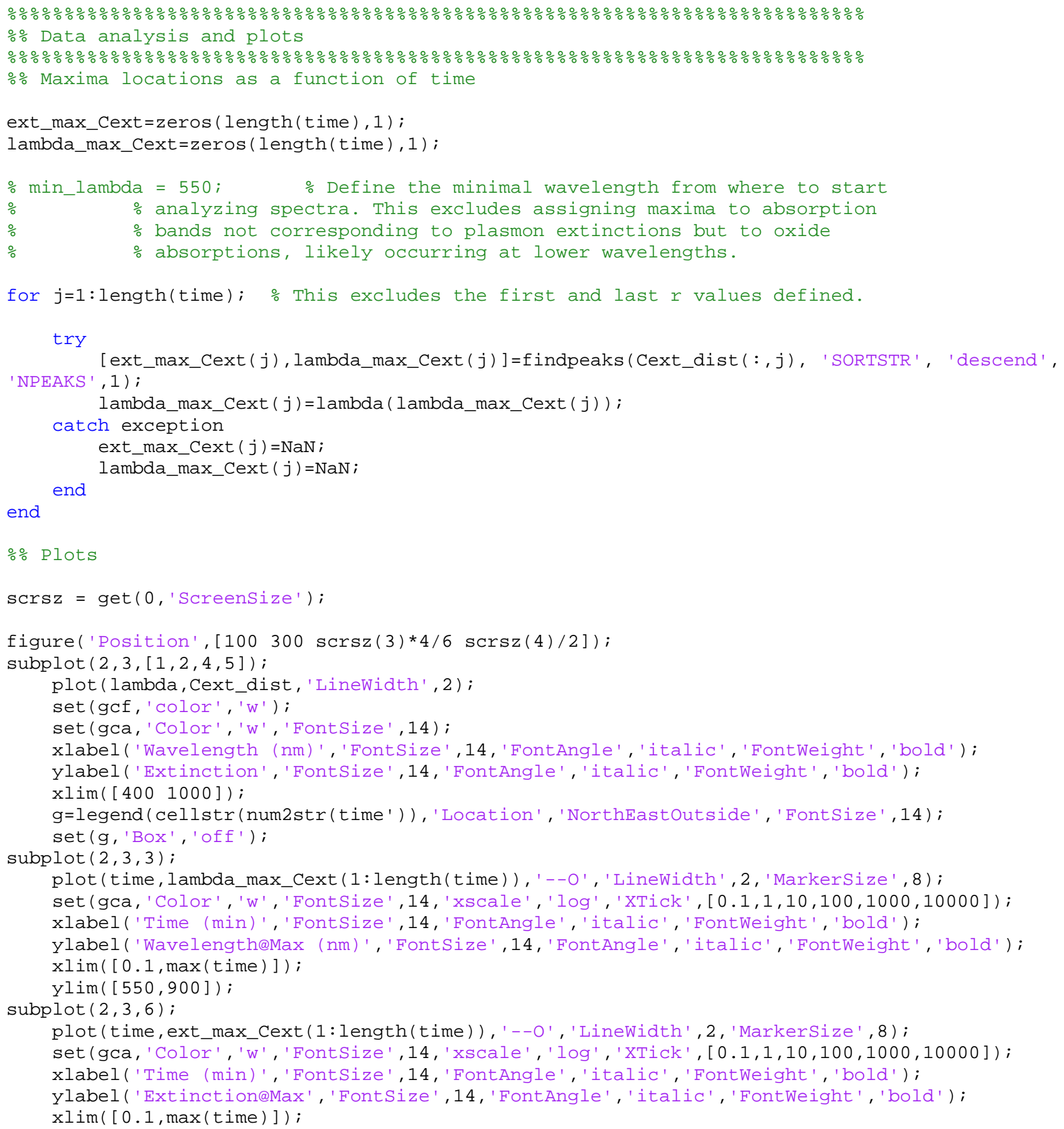

